\title{
Editorial: The Elderly Athlete
}

\author{
Beat Knechtle ${ }^{1,2 *}$, Romuald Lepers ${ }^{3}$, Pantelis Theodoros Nikolaidis ${ }^{4}$ and \\ Caio Victor Sousa ${ }^{5}$
}

${ }^{1}$ Medbase St. Gallen Am Vadianplatz, St. Gallen, Switzerland, ${ }^{2}$ Institute of Primary Care, University of Zurich, Zurich, Switzerland, ${ }^{3}$ CAPS UMR1093 INSERM, Faculty of Sport Sciences, University de Bourgogne-Franche Comté, Dijon, France, ${ }^{4}$ School of Health and Caring Sciences, University of West Attica, Athens, Greece, ${ }^{5}$ Bouve College of Health Sciences, Northeastern University, Boston, MA, United States

Keywords: swimming, running, cycling, endurance, age group

\section{Editorial on the Research Topic}

\section{The Elderly Athlete}

Age-related declines in physical activity levels and exercise training can accelerate the reductions in physical function and performance in older adults. Because elderly athletes (masters athletes) maintain high levels of physical activity, they may serve as an example of an optimal healthy human aging. Indeed, the master athlete has been proposed as an ideal model to determine successful aging due to his or her chronic participation in high-intensity exercise (Lazarus and Harridge, 2017; Tanaka et al., 2019).

The last decades had a remarkable increase in endurance and ultra-endurance events, reflecting a significant participation increase of master athletes. Master athletes compete not only for the glory of participation but also for the achievement of high performance.

In recent years, several astonishing reports show the outstanding achievements of master athletes (Lepers and Stapley, 2016). For example, master athletes compete in distance running (i.e., $100,200,400,800,1,500,5,000,10,000 \mathrm{~m}$, and marathon) until the age group 95-99 years (Schneider et al., 2019). The number of master marathoners older than 75 years has increased in the last 25 years (Ahmadyar et al., 2016), and master marathoners can finish a marathon at the age of 90 years and older (Knechtle et al., 2014a; Mueller et al., 2014). In some instances, male master marathoners older than 90 years can complete a 6-h-run (Knechtle and Nikolaidis, 2018) or even a 12-h-run (Knechtle et al., 2018). Recently, it has been reported that master runners older than 80 or 90 years finished a 100-km ultra-marathon (Stohr et al., 2021).

It is even possible to achieve a World Record after the 100th year of age. A study by Lepers et al. (2016) investigated all best performances achieved by centenarians for swimming, cycling, and running. They found 60 performances belonging to 19 individuals, with 10 in athletics, 8 in swimming, and one in cycling.

Apart from running, swimming seems to be an ideal sports discipline for centenarians. An analysis of participation and performance of male and female age group backstroke swimmers competing in 50, 100, and $200 \mathrm{~m}$ pool swimming at the FINA World Masters Championships held between 1986 and 2014 showed that swimmers in age group 100-104 compete in this discipline (Unterweger et al., 2016). The Canadian Jaring Timmerman, the world's oldest masters swimmer, set four world records in the age group 100-104 years and stands solely responsible for creating the age group 105-109 years in masters swimming (Swam).

In this context, a total of seven articles were published- six studies investigated elderly athletes-in the Research Topic "The Elderly Athlete" (www.frontiersin.org/research-topics/14067/the-elderly-athlete\#overview). These studies investigated different aspects such as muscle strength (Taveira et al.), cardiac characteristics (Wooten et al.; Hoffmann et al.), physical fitness, and lifestyle (Wooten et al.). 
A study comparing trunk muscle strength and postural control of older adults (age $\geq 65$ years old) found thatolder adult runners presented a higher isokinetic torque of extensor trunk muscles postural control than non-runners (Taveira et al.). Two studies explored cardiac aspects of master athletes. A first study investigated sex differences in cardiac structure, function, and left ventricular systolic global longitudinal strain among female and male masters athletes of 55-60 years old, showing cardiac sex differences regarding selected cardiac dimensions (Wooten et al.). A second study compared left ventricular dimensions and function in elite master athletes aged 60 years involved in throwing events requiring strength to those interested in endurance events and sprinting. Left ventricular diastolic function was not different in throwers but superior in endurance athletes and sprinters than age-matched historical controls (Hoffmann et al.). Physical fitness seems to affect also later in life. A study investigating 240 master athletes participating in the World Masters Athletics Championships showed that the lifestyles of master athletes contributed to improved general life satisfaction (Wooten et al.). Another study investigated the age-related decline in running performance of sub-3-h marathoners for five consecutive calendar decades longitudinally. The authors found that it is possible with consistent training and racing regimensto limit the age-related decline in marathon performance to $<7 \%$ per decade at least until 60 years of age (Lepers et al.).

Although athletes of the age of 60-65 years belong by definition to the category of master athletes, athletes at the age of 50 years are at their peak for very long (48-h run) ultramarathons (Knechtle et al., 2014b). Runners older than 70 years

\section{REFERENCES}

Ahmadyar, B., Rosemann, T., Rust, C. A., and Knechtle, B. (2016). Improved race times in marathoners older than 75 years in the last 25 years in the world's largest marathons. Chin. J. Physiol. 59, 139-147. doi: 10.4077/CJP.2016.BAE382

Knechtle, B., Assadi, H., Lepers, R., Rosemann, T., and Rust, C. A. (2014a). Relationship between age and elite marathon race time in world single age records from 5 to 93 years. BMC Sports Sci. Med. Rehabil. 6:31. doi: 10.1186/2052-1847-6-31

Knechtle, B., Jastrzebski, Z., Rosemann, T., and Nikolaidis, P. T. (2018). Pacing during and physiological response after a 12-hour ultra-marathon in a 95-year-old male runner. Front. Physiol. 9:1875. doi: 10.3389/fphys.2018.0 1875

Knechtle, B., and Nikolaidis, P. T. (2018). Pacing in a 94-year-old runner during a 6-hour run. Open Access J. Sports Med. 9, 19-25. doi: 10.2147/OAJSM.S15 5526

Knechtle, B., Valeri, F., Zingg, M. A., Rosemann, T., and Rust, C. A. (2014b). What is the age for the fastest ultra-marathon performance in time-limited races from $6 \mathrm{~h}$ to 10 days? Age 36:9715. doi: 10.1007/s11357-014-9715-3

Lazarus, N. R., and Harridge, S. D. R. (2017). Declining performance of master athletes: silhouettes of the trajectory of healthy human ageing? J. Physiol. 595, 2941-2948. doi: 10.1113/JP27 2443

Lepers, R., and Stapley, P. J. (2016). Master athletes are extending the limits of human endurance. Front. Physiol. 7:613. doi: 10.3389/fphys.2016.00613

Lepers, R., Stapley, P. J., and Cattagni, T. (2016). Centenarian athletes: examples of ultimate human performance? Age Ageing 45, 732-736. doi: 10.1093/ageing/af w111 finishedthe "Leadville 100-Mile Endurance Race" in Colorado, USA (Charles Williams at the age of 70 years), the "Badwater 135Mile Ultramarathon Race" which is considered as the "Toughest Footrace In The World" in California, USA (Jack Deness, 75 years), the "UTMB" (Ultra-Trail du Mont-Blanc) in Chamonix, France (Christoph Geiger, 73 years) or the Western States 100Mile Endurance Run in California, USA (Nick Bassett, 73 years) (Runner, 2019).

Finally, we thank all authors, reviewers, and editors for their contribution to the present Research Topic. We hope that this Research Topic will stimulate further research in this area. Future scientific studies need to investigate the motivation, preparation, and physiology of master athletes 70 years and older up to the centenarians.

To date scientific evidence in the field comes mainly from studies conducted in men only. Research is nevertheless warranted to determine potential sex differences in the effects of lifelong exercise on the age-related decline in performance. The aspect of sex differences in the elderly athlete is a challenging topic especially considering the increase of sports participation in women, e.g., the men-to-women ratio of finishers in the New York City Marathon decreased from 10.16 in the 1970 s to 1.52 in the $2010 \mathrm{~s}$ (Vitti et al., 2020).

\section{AUTHOR CONTRIBUTIONS}

All authors listed have made a substantial, direct and intellectual contribution to the work, and approved it for publication.

Mueller, S. M., Knechtle, B., Knechtle, P., and Toigo, M. (2014). Physiological alterations after a marathon in the first 90-year-old male finisher: case study. Springerplus 3:608. doi: 10.1186/2193-1801-3-608

Runner, B. (2019). Oldest Finishers of Famous Ultra Races. baldrunner.com. Available online at: https://baldrunner.com/2019/09/13/oldest-finishers-offamous-ultra-races (accessed 2021).

Schneider, A. L., Nikolaidis, P. T., and Knechtle, B. (2019). Improved performance in master runners competing in the european championships between 1978 and 2014. J. Strength Cond. Res. 33, 2559-2569. doi: 10.1519/JSC.000000000000 2548

Stohr, A., Nikolaidis, P. T., Villiger, E., Sousa, C. V., Scheer, V., Hill, L., et al. (2021). An analysis of participation and performance of 2067 100$\mathrm{km}$ ultra-marathons worldwide. Int. J. Environ. Res. Public Health 18:362. doi: 10.3390/ijerph18020362

Swam, S. Jaring Timmerman - Oldest Masters Swimmer Passes Away. swimswam.com. Available online at: https://swimswam.com/jaringtimmerman-oldest-masters-swimmer-passes-away-105 (accessed 2021).

Tanaka, H., Tarumi, T., and Rittweger, J. (2019). Aging and physiological lessons from master athletes. Compr. Physiol. 10, 261-296. doi: 10.1002/cphy.c18 0041

Unterweger, C. M., Knechtle, B., Nikolaidis, P. T., Rosemann, T., and Rust, C. A. (2016). Increased participation and improved performance in age group backstroke master swimmers from 2529 to 100-104 years at the FINA world masters championships from 1986 to 2014. Springerplus 5:645. doi: 10.1186/s40064-016-2 209-2

Vitti, A., Nikolaidis, P. T., Villiger, E., Onywera, V., and Knechtle, B. (2020). The "New York City Marathon": participation and 


\section{performance trends of $1.2 \mathrm{M}$ runners during half-century. Res. Sports Med. 28, 121-137. doi: 10.1080/15438627.2019.158 6705}

Conflict of Interest: The authors declare that the research was conducted in the absence of any commercial or financial relationships that could be construed as a potential conflict of interest.
Copyright (c) 2021 Knechtle, Lepers, Nikolaidis and Sousa. This is an open-access article distributed under the terms of the Creative Commons Attribution License (CC $B Y)$. The use, distribution or reproduction in other forums is permitted, provided the original author(s) and the copyright owner(s) are credited and that the original publication in this journal is cited, in accordance with accepted academic practice. No use, distribution or reproduction is permitted which does not comply with these terms. 